\title{
Warum Mikroorganismen Naturstoffe produzieren
}

\author{
MARIO K. C. KRESPACH ${ }^{1,2}$, MARIA C. STROE ${ }^{1}$, AXEL A. BRAKHAGE ${ }^{1,2}$ \\ 1 ABTEILUNG MOLEKULARE UND ANGEWANDTE MIKROBIOLOGIE, LEIBNIZ-INSTITUT \\ FÜR NATURSTOFF-FORSCHUNG UND INFEKTIONSBIOLOGIE - HANS-KNÖLL-INSTITUT, \\ JENA \\ 2 ABTEILUNG MIKROBIOLOGIE UND MOLEKULARBIOLOGIE, INSTITUT FÜR MIKROBIO- \\ LOGIE, UNIVERSITÄT JENA
}

\section{A key role in the communication between fungi and bacteria is played by natural products. Many of their encoding gene clusters are silent under standard laboratory conditions. Interspecies "talk" between microorganisms represents an ecological trigger to activate such silent gene clusters and leads to the formation of novel natural products by the involved species. The understanding of both the activation of silent gene clusters and the ecological function of the produced compounds is of importance to reveal functional microbial interactions required to shape microbiomes.}

DOI: $10.1007 / \mathrm{s} 12268-020-1483-2$

(c) Die Autoren 2020

In Böden, Meeren oder Süßwasserhabitaten leben Mikroorganismen in enger Nachbarschaft mit einer großen Zahl anderer Organismen. Beinahe täglich werden neue Daten zu Mikrobiomen in den verschiedenen Habitaten veröffentlicht, welche die große Diversität der Mikroorganismen eindrucksvoll belegen. In der Regel beantworten diese Studien aber nicht die Fragen, warum bestimmte Mikroorganismen Bestandteil eines definierten Konsortiums sind und was die Zusammensetzung bestimmt. Mikroorganismen haben dafür „Sprachen“ entwickelt, mit denen sie kommunizieren und sich gegenseitig beeinflussen können. Die Sprache der Mikroorganismen besteht größtenteils aus ausgetauschten Molekülen [1]. Eine besondere Rolle dabei spielen Naturstoffe niedermolekulare organische Verbindungen, die viele Mikroorganismen bilden [2]. Einige dieser Substanzen wirken als Antibiotika hemmend auf andere Mikroorganismen. Für fast alle diese Substanzen ist allerdings ihre ökologische Funktion im Habitat unbekannt [3]. Die Aufklärung ihrer Funktionen ist wichtig, um generelle Prinzipien der Interaktion von Mikroorganismen verstehen zu lernen.
Bakterien wie Streptomyzeten oder filamentöse Pilze wie Aspergillus-Spezies können mehr als 80 Biosynthesegencluster besitzen, von denen viele in Monokulturen der Mikroorganismen nicht aktiv sind. Erst der ökologische Kontext, etwa die Anwesenheit anderer Mikroorganismen, führt oftmals zur Aktivierung der Gencluster und damit der Produktion von Naturstoffen, die dann ihre Wirkung entfalten können.

\section{Bodenbakterien aktivieren die} Produktion von Naturstoffen in Pilzen Um die Aktivierung von Naturstoff-Biosynthesen zu erforschen, kokultiviert unsere Arbeitsgruppe bodenlebende Pilze und Bakterien. Dabei entdeckten wir, dass das Bakterium Streptomyces rapamycinicus im filamentösen Modellpilz Aspergillus nidulans ein stilles Gencluster aktiviert [4]. Bei dem Cluster handelt es sich um das ors-Biosynthesegencluster, dessen Aktivierung zur Biosynthese von Orsellinsäure und deren Derivaten führt (Abb. 1). Interessanterweise beschränkt sich die Fähigkeit zur Aktivierung dieser Naturstoffe nur auf einige nah verwandte Arten bekannter Streptomyceten. Diese Beobachtung legte den Grundstein für die systematische Analyse von zunächst binären Interaktionssystemen.

In folgenden Untersuchungen konnten wir zeigen, dass $S$. rapamycinicus in der Lage ist, auch in Aspergillus fumigatus, einem wichtigen humanpathogenen Pilz, inaktive Biosynthesegencluster zu aktivieren, was zur Produktion der Fumicycline [5] und Fumigermine [6] führt (Abb. 1). Fumigermine inhibieren die Auskeimung der Sporen des induzierenden Bakteriums S. rapamycinicus. Die Substanzen helfen somit dem Pilz, die Ressourcen des gemeinsamen Habitats gegen einen bakteriellen Konkurrenten zu verteidigen.

A. fumigatus reagiert auch auf weitere Streptomyceten mit der Produktion von Naturstoffen. In Anwesenheit von S. peuceticus produziert A. fumigatus einen Cocktail verschiedener Naturstoffe, die zwar nicht antibakteriell, aber zum Teil zytotoxisch wirken [7]. S. bullii löst in A. fumigatus die Produktion von neuen Naturstoffen (Fumitremorgin B, Spirotryprostatin A) aus, die gegen die Erreger der Chagas-Krankheit, Trypanosoma bruzei, und der Leishmaniose, Leishmania donovani, wirken (Abb. 1, [8]).

Die Sprache verstehen: S. rapamycinicus modifiziert Pilz-Chromatin

Als sich herausstellte, dass Bakterien die Produktion von Naturstoffen in Pilzen auslösen können, war es naheliegend zu erforschen, wie das Bakterium in die Genregulation im Pilz eingreift. Weitergehende Untersuchungen der Interaktion zwischen S. rapamycinicus und A. nidulans und der Induktion des ors-Genclusters führten zu der Erkenntnis, dass das Bakterium in der Lage ist, epigenetisch wirksame Histonmodifikationen im Pilz zu verändern (Abb. 2). In Eukaryoten ist die DNA um Histonkomplexe gewickelt. Im kondensierten Heterochromatin ist die Expression der DNA weitgehend blockiert. Die für die Expression nötige Auflockerung der DNA zum Euchromatin erfordert die Modifikation von Histonen, z. B. durch Acetylierung. Die Anwesenheit von Streptomyceten bewirkt nun die verstärkte Acetylie- 

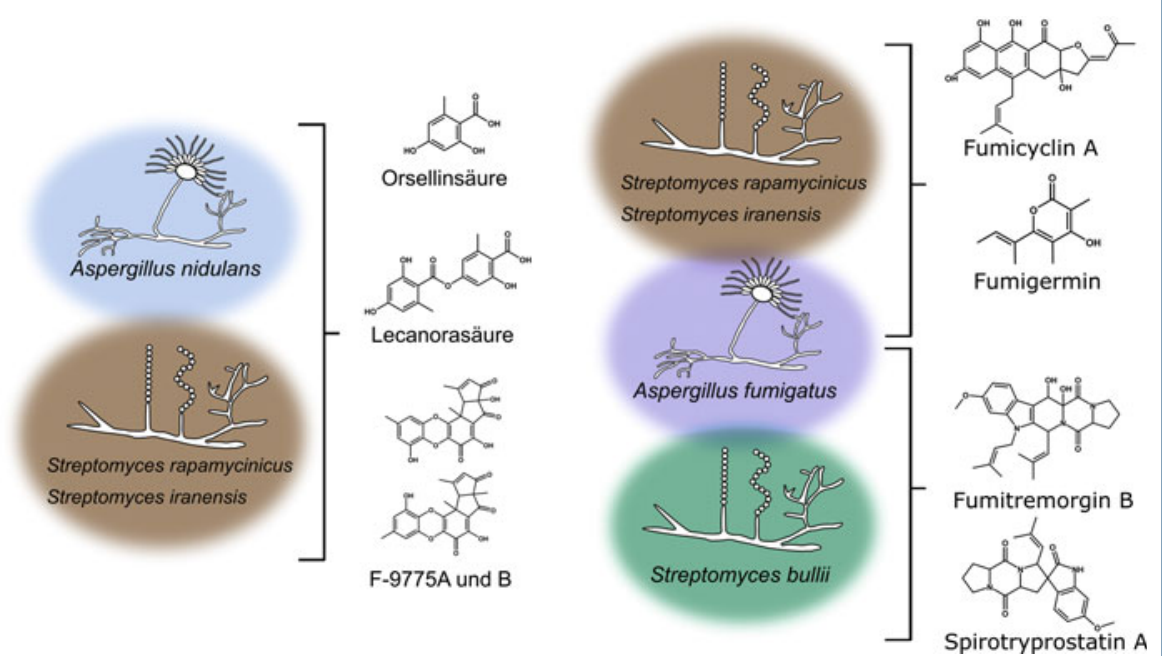

$\Delta$ Abb. 1: Übersicht mikrobieller Interaktionen und dadurch produzierter Metabolite. Während das Bakterium Streptomyces rapamycinicus im Pilz Aspergillus nidulans die Produktion von Orsellinsäure, Lecanorasäure und Derivaten aktiviert, reagiert $A$. fumigatus auf dieses Bakterium mit der Produktion von Fumicyclin A und Fumigermin. S. bullii aktiviert die Produktion von Fumitremorgin B und Spirotryprostatin A in A. fumigatus.

Abb. 2: Aktivierung des ors-Genclusters im Pilz Aspergillus nidulans durch das Bakterium Streptomyces rapamycinicus. Das Bakterium überträgt ein bisher unbekanntes Signal, das zur Aktivierung des GcnEKomplexes führt. Dieser ermöglicht durch Auflockerung der Chromatinstruktur die Transkription des ors-Clusters und des basR-Gens. BasR ermöglicht die Transkription des orsClusters, das Orsellinsäure und dessen Derivate produziert.

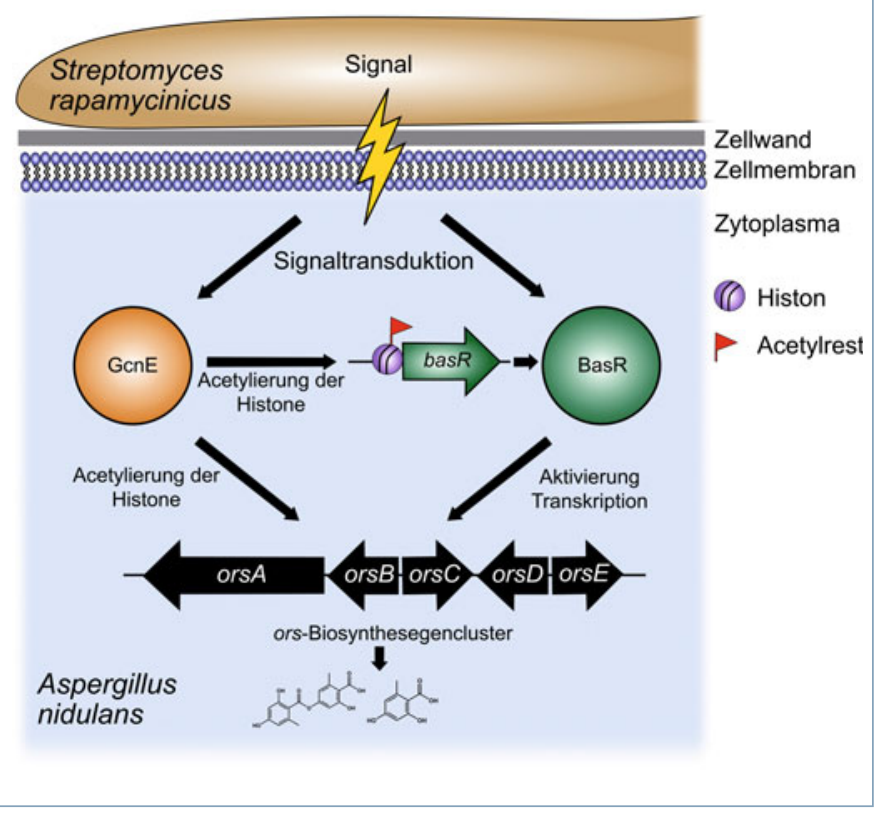

rung bestimmter Lysin-Reste im Histon H3, was zur Auflockerung der DNA speziell in der Region des ors-Biosynthesegenclusters führt, sodass dieses abgelesen werden kann (Abb. 2, [9]). Verantwortlich für die Acetylierung ist die pilzliche Lysin(Histon)-Acetyltransferase GcnE.

Mittels einer genomweiten ChIP-Seq-Analyse fanden wir weitere Bereiche der DNA, die bei der Kokultivierung von Pilz und Bakterium aufgelockert werden. Dazu zählt ein Gen, das den Regulator BasR codiert [10]. Dieser ist für die Aktivierung des ors-Biosynthesegenclusters essenziell. BasR ist also ein zentraler Schalter des Pilzes, den S. rapamycinicus anzuschalten vermag.

\section{Dieselbe Sprache sprechen: BasR sagt Pilz-Interaktionspartner vorher}

Die Information von BasR als Schlüsselregulator nutzten wir, um weitere Pilze zu identifizieren, deren Genom diesen Regulator und zugleich das ors-Gencluster codieren. Einer dieser Pilze ist $A$. sydowii. Die spannende Frage war nun, ob die Anwesenheit von BasR und des ors-Genclusters ausreicht, um das ors-Cluster auch durch S. rapamycinicus zu induzieren [10]. Das ist in der Tat der Fall.
S. rapamycinicus induziert auch in $A$. sydowii das ors-Gencluster. Wir vermuten, dass in Zukunft aufgrund des genetischen Inventars von Mikroorganismen deren Interaktionspartner vorhergesagt werden können.

\section{Von der bipartiten zur tripartiten Interaktion}

Um funktionelle Zusammenhänge in mikrobiellen Gemeinschaften erkennen zu können, ist die Bildung komplexerer Interaktionssysteme in vitro hilfreich. Deshalb versuchten wir, weitere Interaktionspartner des bipartiten Systems aus S. rapamycinicus/A. nidulans zu entdecken. Wie sich herausstellte, interagiert auch die einzellige Grünalge Chlamydomonas reinhardtii funktionell mit beiden Partnern. Vorherige Arbeiten hatten bereits belegt, dass in A. nidulans sowie C. reinhardtii die Fähigkeit zur Bildung einer mutualistischen Symbiose immer noch besteht [11]. Interessanterweise besitzt diese AlgenPilz-Interaktion auch eine funktionale Bedeutung für die Interaktion mit dem dritten Partner, S. iranensis (Abb. 3). S. iranensis ist ein naher Verwandter von $S$. rapamycinicus und aktiviert auch das ors-Biosynthesegencluster in A. nidulans. Kokultiviert man nun das Bakterium mit $C$. reinhardtii, stirbt die Alge [12]. Der Grund dafür ist das Algizid Azalomycin F, das S. iranensis bei Kontakt mit C. reinhardtii sekretiert. Allerdings hilft $C$. reinhardtii die Interaktion mit $A$. nidulans: C. reinhardtii wird von $A$. nidulans angelockt und schwimmt aktiv auf den Pilz zu. C. reinhardtii-Zellen, die sich im Pilzmyzel befinden, sind vor der Wirkung von Azalomycin F geschützt. Dieser Schutzeffekt basiert darauf, dass Azalomycin F an polare Membranlipide bindet und so A. nidulans Azalomycin F durch seine große Biomasse und Oberfläche abfangen kann. Dieses Beispiel zeigt, wie sich durch das Erweitern eines bipartiten mikrobiellen Systems durch einen weiteren Partner die Komplexität erhöht und wie eine temporäre „Symbiose“ einen der Partner vor einem eindringenden bakteriellen Gegner schützt.

\section{Wie Naturstoffe Mikrobiome strukturieren und verändern}

Für ein besseres Verständnis der Strukturierung von Mikrobiomen und der Funktion von Naturstoffen für diesen Prozess ist die Rekonstitution von mikrobiellen Interaktionssystemen von enormer Bedeutung. Diese bestehen zumeist zu Beginn nur aus zwei interagierenden Partnern, können dann aber um weitere 


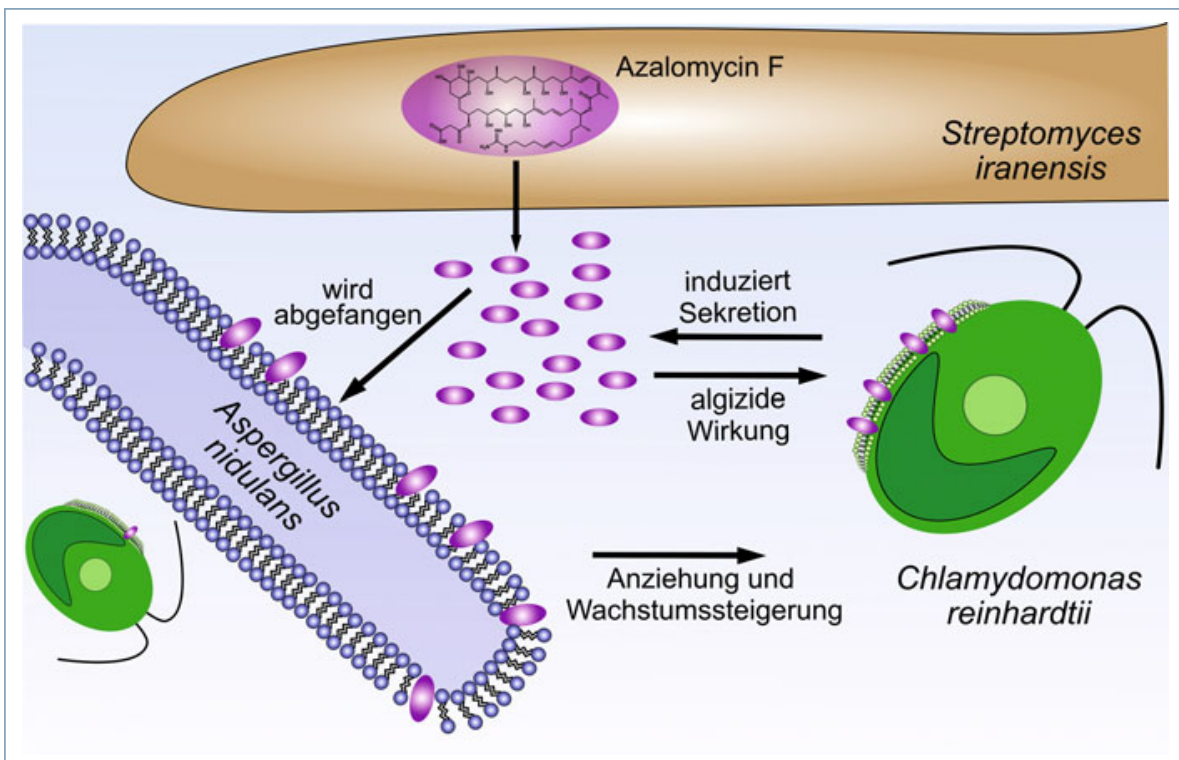

A Abb.3: Die tripartite Interaktion des Bakteriums Streptomyces iranensis, des Pilzes Aspergillus nidulans und der Alge Chlamydomonas reinhardtii. Die Alge induziert die Sekretion von Azalomycin $\mathrm{F}$ durch $S$. iranensis. Werden $C$. reinhardtii und $A$. nidulans kokultiviert, lagert sich die Alge in das Pilzgeflecht ein. Die Alge ist geschützt, da Azalomycin F größtenteils von der Cytoplasmamembran von $A$. nidulans abgefangen wird. Verändert aus [12].

Organismen erweitert werden, um stabile, sich in einem dynamischen Gleichgewicht befindliche mikrobielle Konsortien zu etablieren. Eine Schlüsselrolle spielen dabei Naturstoffe, deren Produktion und Bedeutung häufig vom ökologischen Kontext und insbesondere von Partnermikroorganismen abhängen. In Zukunft wird es von großem Interesse sein, die Bedeutung dieser Naturstoffe als Sprache weiter zu verstehen.

Vermutlich werden durch die Entdeckung grundlegender Prinzipien der Auslösung und der Produktion von Naturstoffen bei der Interaktion von Mikroorganismen neue Wirkstoffe gefunden sowie gezielte Vorhersagen von Interaktionspartnern möglich.

\section{Danksagung}

Wir bedanken uns bei den Mitarbeitern beider Institute und die exzellenten Kooperationen zwischen den verschiedenen Gruppen unserer Forschungsprogramme. Diese Arbeit wurde durch den DFG-SFB ChemBioSys, das DFG-Exzellenzcluster Balance of the Microverse und das BMBF-Programm InfectControl unterstützt.

\section{Literatur}

[1] Mithöfer A, Boland W (2016) Do you speak chemistry? EMBO Rep 17:626-629

[2] Brakhage AA (2013) Regulation of fungal secondary metabolism. Nat Rev Microbiol 11:21-32

[3] Macheleidt J, Mattern DJ, Fischer J et al. (2016)

Regulation and role of fungal secondary metabolites. Annu Rev Genet 50:371-392
[4] Schroeckh V, Scherlach K, Nützmann HW et al. (2009) Intimate bacterial-fungal interaction triggers biosynthesis of archetypal polyketides in Aspergillus nidulans. PNAS 106:14558-14563

[5] König CC, Scherlach K, Schroeckh V et al. (2013) Bacterium induces cryptic meroterpenoid pathway in the pathogenic fungus Aspergillus fumigatus. ChemBioChem 14:938-942

[6] Stroe MC, Netzker T, Scherlach K et al. (2020) Targeted induction of a silent fungal gene cluster encoding the bacteria-specific germination inhibitor fumigermin. eLife 9:e5254 [7] Zuck KM, Shipley S, Newman DJ (2011) Induced production of N-formyl alkaloids from Aspergillus fumigatus by

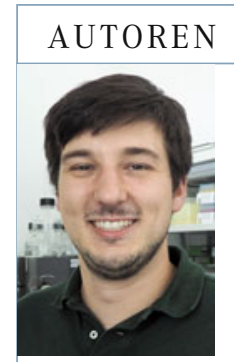

Mario Krespach

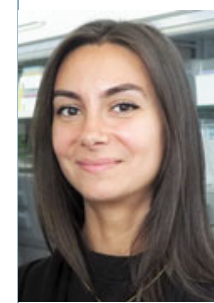

Maria Stroe Gruppe.

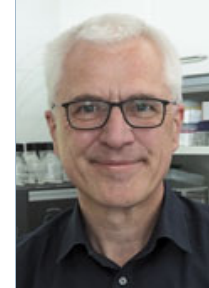

Axel Brakhage und Vizepräsident der DFG. co-culture with Streptomyces peucetius. J Nat Prod 74:16531657

[8] Rateb ME, Hallyburton I, Houssen WE et al. (2013) Induction of diverse secondary metabolites in Aspergillus fumigatus by microbial co-culture. RSC Adv 3:14444-14450 [9] Nützmann H-W, Reyes-Dominguez Y, Scherlach K et al. (2011) Bacteria-induced natural product formation in the fungus Aspergillus nidulans requires Saga/Ada-mediated histone acetylation. PNAS 108:14282-14287

[10] Fischer J, Müller SY, Netzker T et al. (2018) Chromatin mapping identifies BasR, a key regulator of bacteria-triggered production of fungal secondary metabolites. eLife 7:e40969 [11] Hom EFY, Murray AW (2014) Niche engineering demonstrates a latent capacity for fungal-algal mutualism. Science 345:94-98

[12] Krespach MKC, García-Altares M, Flak M et al. (2020) Lichen-like association of Chlamydomonas reinhardtii and Aspergillus nidulans protects algal cells from bacteria. ISME J 14:2794-2805

Funding note: Open Access funding enabled and organized by Projekt DEAL. Funding note: Open Access funding enabled and organized by Projekt DEAL.
Open Access: Dieser Artikel wird unter der Creative Commons Namensnennung 4.0 International Lizenzveröffentlicht, welche die Nutzung Vervielfälttigung. Bearbeitung Verbreitung und Wiedergabe in jeglichem Medium und Format erlaubt, sofern Sie den/die ursprünglichen Autor(en) und die Quelle ordnungsgemäß nennen, einen Link zur Creative Commons Lizenz beifügen und angeben, ob Änderungen vorgenommen wurden. Die in diesem Artikel enthaltenen Bilder und sonstiges Drittmaterial unterliegen ebenfalls der genannten Creative Commons Lizenz, sofern sich aus der Abbildungslegende nichts anderes ergibt. Sofern das betreffende Material nicht unter der genannten Creative Commons Lizenz steht und die betreffende Handlung nich hach gesetzlichen Vorschriten erlaubt ist, ist fur die oben aufgetuhrten gung des jeweiligen Lizenzinformation auf http://creativecommons org/licenses/by/4.0/deder

Korrespondenzadresse:

Prof. Dr. Axel A. Brakhage

Leibniz Institut für Naturstoff-Forschung und Infektionsbiologie (Leibniz-HKI)

Adolf-Reichwein-Straße 23

D-07745 Jena

Institut für Mikrobiologie

Friedrich-Schiller-Universität Jena

Neugasse 23

D-07743 Jena

axel.brakhage@leibniz-hki.de

Jahrgang 1989. 2010-2013 Biologiestudium (Bachelor) an der Universität Tübingen.

2013-2016 Mikrobiologiestudium (Master) an der Universität Jena. Seit 2016 Doktorand in der Arbeitsgruppe von Prof. Dr. A. Brakhage.

Jahrgang 1991. 2010-2014 Integrated Master's degree in „Chemistry with Pharmacology“ an der Universität Birmingham, UK. 2014-2019 Promotion an der Universität Jena in der Arbeitsgruppe von Prof. Dr. A. Brakhage. Seit 2020 Postdoc in derselben

Seit 2004 am Lehrstuhl für Mikrobiologie und Molekularbiologie, Institut für Mikrobiologie, Universität Jena. Seit 2005 Direktor, Leibniz-Institut für Naturstoff-Forschung und Infektionsbiologie (Leibniz-HKI) und Leiter der Abteilung Molekulare und Angewandte Mikrobiologie. Sprecher des Exzellenzclusters Balance of the Microverse, des BMBF-Konsortiums InfectControl sowie des SFB/TR Human-pathogene Pilze und ihr menschlicher Wirt - Netzwerke der Interaktion (FungiNet), Senator der Leopoldina 